\title{
Metodologia de Aquisição e Processamento em Dados de Georradar (GPR) nos Depósitos Quaternários da Porção Emersa da Bacia de Pelotas
}

\author{
Eduardo Guimarães Barboza1, Maria Luiza Correa da Camara Rosa ${ }^{1}$, Felipe Caron ${ }^{2}$ \\ 1 Instituto de Geociências (IGEO) - Universidade Federal do Rio Grande do Sul (UFRGS), RS, Brasil. \\ 2 Universidade Federal do Pampa (UNIPAMPA), Campus Caçapava do Sul, RS, Brasil.
}

Copyright 2014, SBGf - Sociedade Brasileira de Geofísica

Este texto foi preparado para a apresentação no VI Simpósio Brasileiro de Geofísica, Porto Alegre, 14 a 16 de outubro de 2014. Seu conteúdo foi revisado pelo Comitê Técnico do VI SimBGf, mas não necessariamente representa a opinião da SBGf ou de seus associados. É proibida a reprodução total ou parcial deste material para propósitos comerciais sem prévia autorização da SBGf.

\section{Resumo}

Dados provenientes do método geofísico eletromagnético utilizado no sistema de Georradar (GPR) são aplicados há mais de dez anos nos estudos realizados nos sistemas deposicionais costeiros quaternários da Bacia de Pelotas. Esses estudos possuem como principal objetivo caracterizar a evolução costeira ao longo da porção emersa da Bacia de Pelotas. Métodos de aquisição e processamento dos dados obtidos durante o desenvolvimento destes estudos propiciam, atualmente, um fluxograma de coleta de dados otimizado, produzindo excelentes resultados para a interpretação dos processos que resultaram na atual configuração da planície costeira, componente geomorfológica da expressão continental da referida bacia.

\section{Introdução}

A planície costeira ao longo da Bacia de Pelotas corresponde a sua porção continental emersa, e é constituída por sistemas de leques aluviais, que afloram em sua porção oeste, e por quatro sistemas deposicionais do tipo laguna-barreira (Fig. 1). Conforme Villwock \& Tomazelli (1995), esses sistemas se desenvolveram a partir de ciclos transgressivosregressivos controlados pela glacioeustasia e registram máximos transgressivos seguidos por regressões. De acordo com a idade, para cada sistema laguna-barreira foi estabelecido um número, sendo a Barreira I a mais antiga e a Barreira IV a mais jovem, ainda ativa.

O registro estratigráfico em subsuperfície destes sistemas obteve um grande avanço no conhecimento através do método geofísico do Georradar (GPR). Pois, até meados do final do século passado dados de subsuperfície para esta área da bacia eram obtidos por amostragens pontuais através de dados de testemunhos de sondagens.

Os estudos foram desenvolvidos desde os sistemas lagunares até os sistemas praias, abrangendo depósitos pleistocênicos e holocênicos ao longo da bacia. Diversos trabalhos foram realizados desde o sul até o norte da bacia, destacando-se: no Cabo Polônio (Barboza et al., 2012), no Hermenegildo (Barboza et al., 2011; Caron, 2014; Caron et al., 2010, 2011, 2014; Lima et al., 2013; Rosa, 2012), em Santa Vitória do Palmar (Rosa, 2012), em Curral Alto (Watanabe et al., 2013), no Taim (Tomazelli et al., 2008), no Farol Sarita (Caron et al., 2014), no Cassino (Barboza et al., 2011; Rosa, 2012), no Estreito (Barboza et al., 2014b); em Turuçu (Manzolli et al., 2010; Salgado et al., 2013), no Bujuru (Rosa, 2012; Dillenburg \& Barboza, 2014), em Mostardas (Barboza, 1999; Barboza et al., 1999), em Dunas Altas (Becker et al., 2008; Barboza et al., 2011), no Jardim do Éden (Rosa, 2012; Rosa et al., 2012), em Osório (Barboza et al., 2008; Fracalossi et al., 2010), em Terra de Areia (Rosa, 2012; Bitencourt et al., 2013, 2014), em Curumim (Barboza et al., 2009, 2010, 2011, 2013), em Torres (Rockett et al., 2013, 2014), em Passo de Torres (Barboza et al., 2014a; Biancini da Silva et al., 2010, 2013, 2014), em Campo Bom (Oliveira \& Barboza, 2014), no Camacho (Fornari et al., 2012).

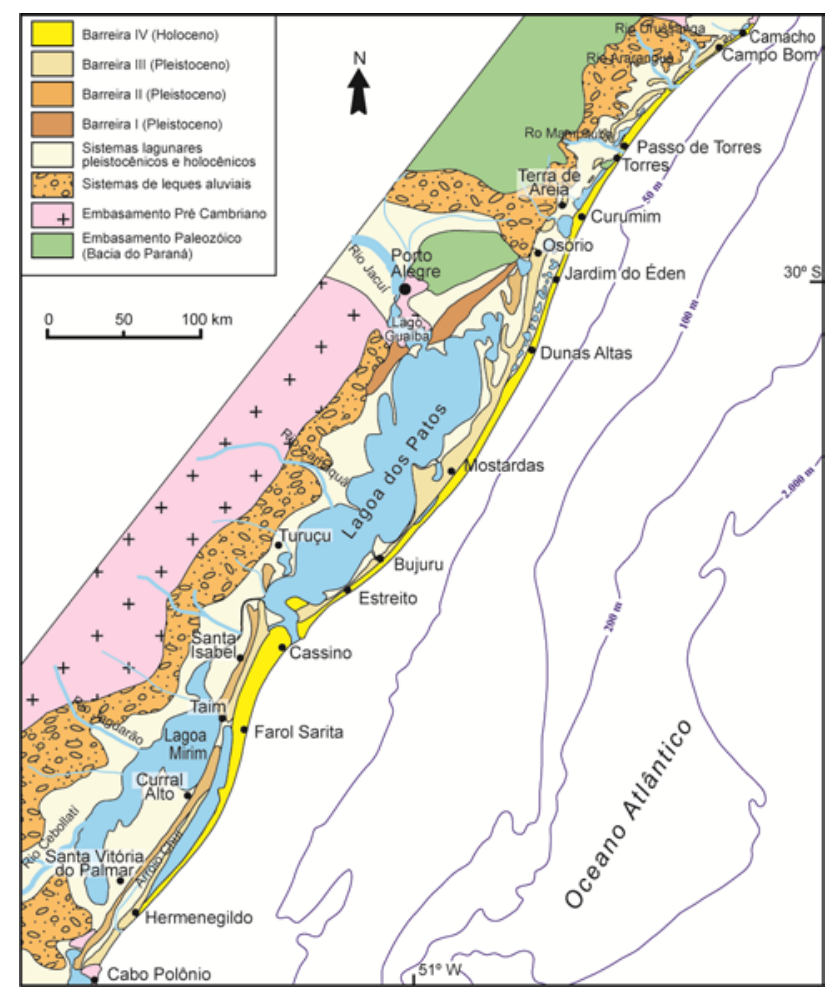

Figura 1 - Mapa geológico/geomorfológico da Planície Costeira da Bacia de Pelotas entre La Coronilla e Camacho (modificado de Dillenburg \& Barboza, 2014).

Técnicas de obtenção de dados com o Georradar evoluíram tanto nos equipamentos utilizados, quanto nos métodos de aquisição e processamento. Assim, os resultados obtidos nas áreas supracitadas apresentam 
um registro fidedigno da geometria e estrutura interna dos depósitos costeiros, os quais auxiliam no refinamento dos modelos geológicos.

\section{Metodologia}

A primeira etapa consiste na aquisição das seções através no modo Common Offset. Este método é empregado através do deslocamento simultâneo e no mesmo sentido das antenas transmissora e receptora, posicionadas a uma distância constante (fixed offset).

Durante o levantamento diversos parâmetros devem ser selecionados de acordo com os objetivos e com os tipos de alvos a serem identificados. A distância entre os pontos de amostragem (resolução horizontal - step size), a profundidade da janela de aquisição, a separação entre as antenas, o empilhamento dos traços (stacking) a orientação das antenas, a localização e o espaçamento entre as linhas são alguns desses parâmetros (Jol \& Bristol, 2003). Além disso, outra importante questão a ser considerada durante a aquisição relaciona-se com o posicionamento espacial das seções, principalmente em áreas de relevo acidentado, onde é necessária a realização de correções topográficas.

A segunda etapa do trabalho é a de processamento das seções. Uma seção de Georradar pode ser comparada a um afloramento, contendo feições relacionadas com a gênese e com a alteração natural e/ou antrópica das rochas (Fig. 2). O que define os métodos de processamento a serem utilizados são os objetivos e os tipos de feições que se desejam identificar. Em levantamentos para engenharia, o interesse pode estar na localização de dutos e outros alvos de origem antrópica. Dessa forma, os processamentos não poderão ser os mesmos aplicados em estudos geológicos, em que muitas vezes, esses alvos são removidos do registro.

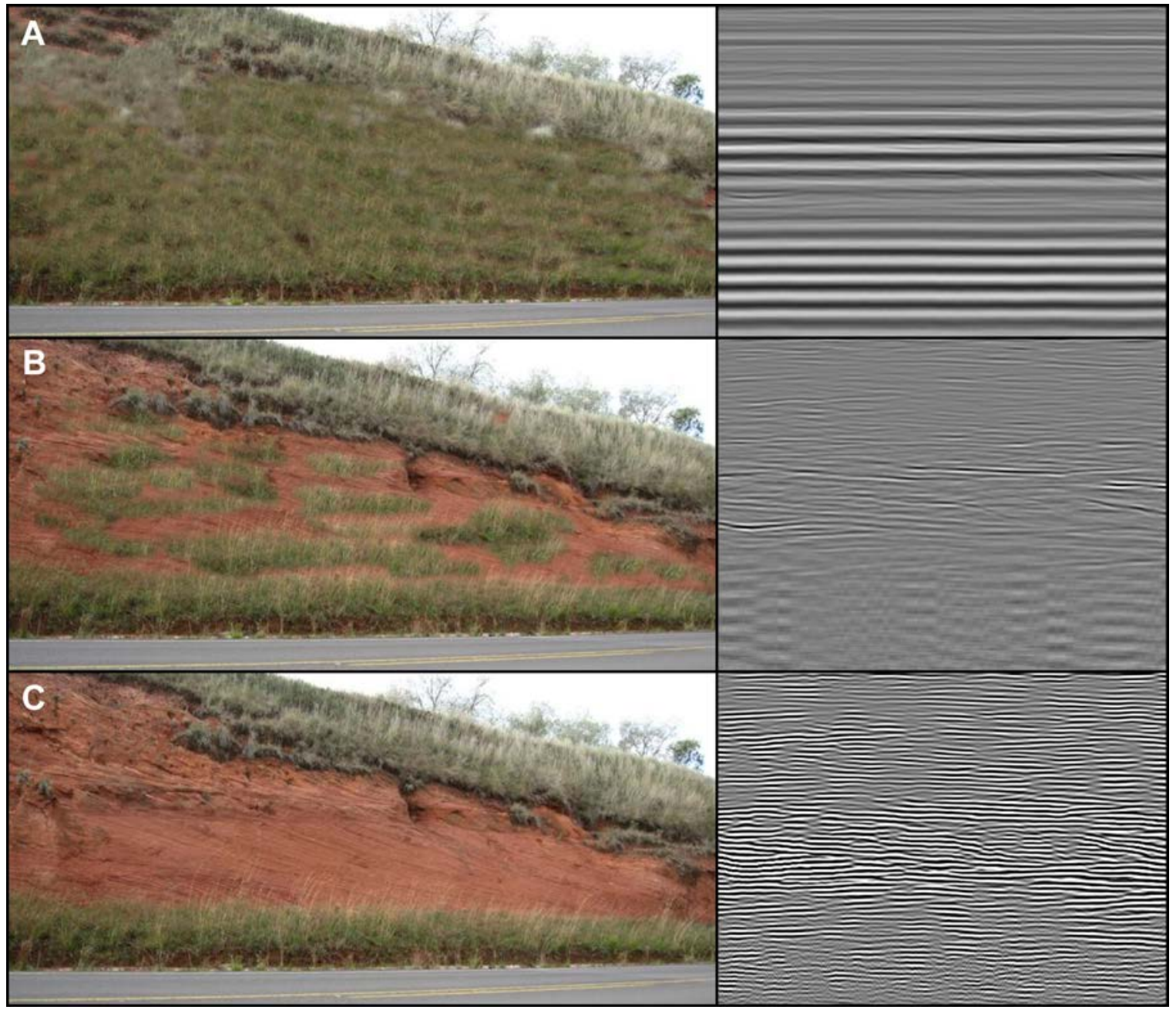

Figura 2 - Analogia entre o processamento de seções de Georradar com a limpeza de um afloramento rochoso. A) As feições geológicas da seção encontram-se mascaradas por ondas aéreas e da superfície do terreno, que aparecem como refletores plano-paralelos. B) Na seção, um primeiro processamento remove os refletores plano-paralelos, permitindo a visualização de alguns refletores relacionados aos depósitos sedimentares. C) Finalmente, a aplicação de filtros de frequência e ganhos ressalta os refletores, deixando a seção pronta para a interpretação (modificado de Rosa, 2012). 


\section{Equipamentos}

Os coletores que vêm sendo utilizados nas aquisições são o modelo SIR-3000 da empresa GSSI ${ }^{\mathrm{TM}}$ (Geophysical Survey Systems, Inc.), e o Cobra Plug-In TM (Radarteam Sweden AB), sistema sem fios utilizado com as antenas aéreas do mesmo fabricante. São empregadas para a aquisição antenas de contato nas frequências de 100, 200, 270 e $400 \mathrm{MHz}$, fabricadas pela GSSI ${ }^{\mathrm{TM}}$, as quais são utilizadas no modo monoestático. Podem também serem utilizadas antenas aéreas nas frequências centrais de 40, 70 e $150 \mathrm{MHz}$, fabricadas pela Radarteam Sweden $A B$, no modo mono ou bi estático acoplado a um módulo de potência PRFPLUS$600^{\mathrm{TM}}$ da empresa Geoscanners AB. Durante a aquisição o sistema de GPR é conectado a um receptor GNSS Topográfico ou Geodésico permitindo o posicionamento das seções através do método diferencial.

\section{Aquisição}

$\mathrm{Na}$ aquisição realizada no modo Common Offset, as antenas podem ser deslocadas manualmente, através de um caminhamento, ou acopladas a um veículo. A aquisição é realizada com as antenas orientadas perpendicularmente ao sentido de deslocamento, a uma velocidade média de $15 \mathrm{~km} / \mathrm{h}$ quando acopladas ao veículo (Fig. 3). A distância entre os pontos de leitura, que define a resolução horizontal, normalmente é de 10 $\mathrm{cm}$. As profundidades das janelas de aquisição, dependendo do alvo, variam de 300 a 1000 ns, que correspondem a profundidades aproximadas entre 15 e $45 \mathrm{~m}$. Cada traço da seção representa o empilhamento de 32 leituras (stacking), aplicando-se um ganho do tipo AGC (Automatic Gain Control) com a finalidade equalizar a amplitude dos traços, que tende a ser atenuada em profundidade. A constante dielétrica utilizada para converter o tempo em profundidade durante os levantamentos, geralmente relacionada com depósitos arenosos, é a de valor 10, que representa uma velocidade de 0,09 m/ns, (Davis \& Annan, 1989).

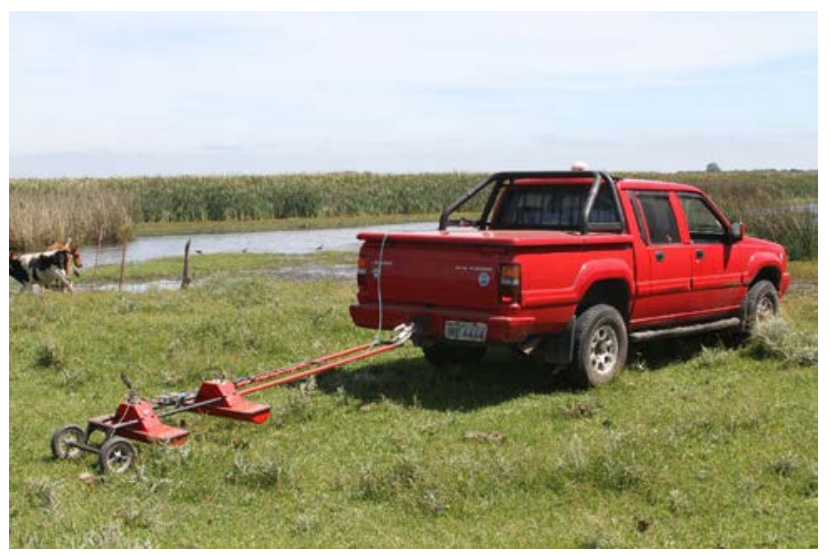

Figura 3 - Arranjo bi estático utilizando antenas aéreas com frequência central de $150 \mathrm{MHz}$ acopladas a um veiculo para aquisição em terrenos sem obstáculos.

\section{Processamentos}

Os processamentos são realizados em três programas computacionais distintos, o Radan ${ }^{\mathrm{TM}}$, o Reflex-Win ${ }^{\circledR}$ e o Prism2 ${ }^{\circledR}$.

A primeira etapa consiste da abertura das seções brutas no programa Radan ${ }^{\mathrm{TM}}$, onde é aplicado um filtro espacial para a remoção de um ruído sistemático relacionado com a onda aérea (background removal). Este ruído deve-se à utilização de antenas aéreas e, em algumas situações com as antenas de contato acopladas ao solo, quando ocorre o desacoplamento do solo das mesmas devido a irregularidades no terreno. Este fenômeno se manifesta na forma de refletores plano-paralelos contínuos que se repetem ao longo de toda a janela da seção (Fig. 4). Devido à presença desses refletores, não é possível observar as reflexões oriundas dos depósitos sedimentares em tempo real.

Em seguida, é aplicado um filtro de frequência (passa banda) para reduzir ruídos aleatórios, cortando frequências mais baixas e mais altas que a frequência central da antena. Um ganho do tipo exponencial pode ser empregado para a ampliação dos sinais atenuados em profundidade, os quais são ainda mais atenuados após a realização de outros processamentos. Esse procedimento suprime a identificação do freático, que pode ser observado em algumas seções como um refletor diácrono com tendência horizontal ou similar a da topografia, acima do qual há uma diminuição na amplitude dos refletores. Com a aplicação do ganho, as amplitudes são equalizadas ao longo das seções. Finalmente, a posição do topo da seção é corrigida, ajustando a profundidade zero com a superfície do terreno.

Após o processamento no $\operatorname{Radan}^{\mathrm{TM}}$, os dados são importados para o programa Reflex-Win ${ }^{\circledR}$ ou Prism $2^{\circledR}$, onde é aplicado um segundo filtro de frequência trapezoidal (Ormsby bandpass). Esse procedimento é realizado devido à remoção muito mais eficiente dos ruídos presentes na seção e à existência de mais possibilidades na determinação dos parâmetros dos filtros, diferentemente dos filtros de frequência existentes no programa Radan ${ }^{\mathrm{TM}}$. Nestes dois programas é possível determinar além dos limites inferior e superior de corte, os limites de um platô, definindo um filtro com forma trapezoidal. Para tanto é necessária a identificação de picos anômalos, os quais são de fácil determinação no espectro de frequências. Assim, é possível estabelecer de maneira precisa os parâmetros que devem ser aplicados neste filtro trapezoidal.

Posteriormente, as seções são exportadas e novamente visualizadas no programa Radan ${ }^{\mathrm{TM}}$, onde se procede com a correção topográfica e, caso seja necessário, é aplicado mais um ganho. A conversão do tempo de viagem da onda para profundidade em metros é realizada através da obtenção de dados físicos proveniente de sondagens ou através do método de levantamento CMP (Common Mid Point), utilizando-se duas antenas. 
Em muitos casos surge a dúvida se o processamento alterou o registro original, ou seja, se os procedimentos criaram refletores inexistentes no dado real. Seguindo o que diz Sangree \& Widmier (1979) se referindo à sísmica, "o intérprete deve aprender a lidar com os problemas, exceções e armadilhas". Para resolver tal questionamento pode ser empregado, quando ocorrem dúvidas, um procedimento inspirado em outra área das Geociências, a Geocronologia. Através do princípio da reprodutibilidade é possível validar os dados com base na sua repetição e reprodução.
Partindo do princípio que os ruídos do ambiente são predominantemente aleatórios e que os dados devem se reproduzir, são analisadas seções adquiridas repetidamente em um mesmo local. Sobre dados oriundos de múltiplos levantamentos, realizados com antenas distintas ou em sentidos opostos sobre o mesmo perfil, são delimitados os principais refletores. Esse procedimento pode ser realizado após um processamento mínimo, realizado no programa Radan ${ }^{\mathrm{TM}}$, contemplando somente a aplicação do filtro espacial (background removal) e do ganho.

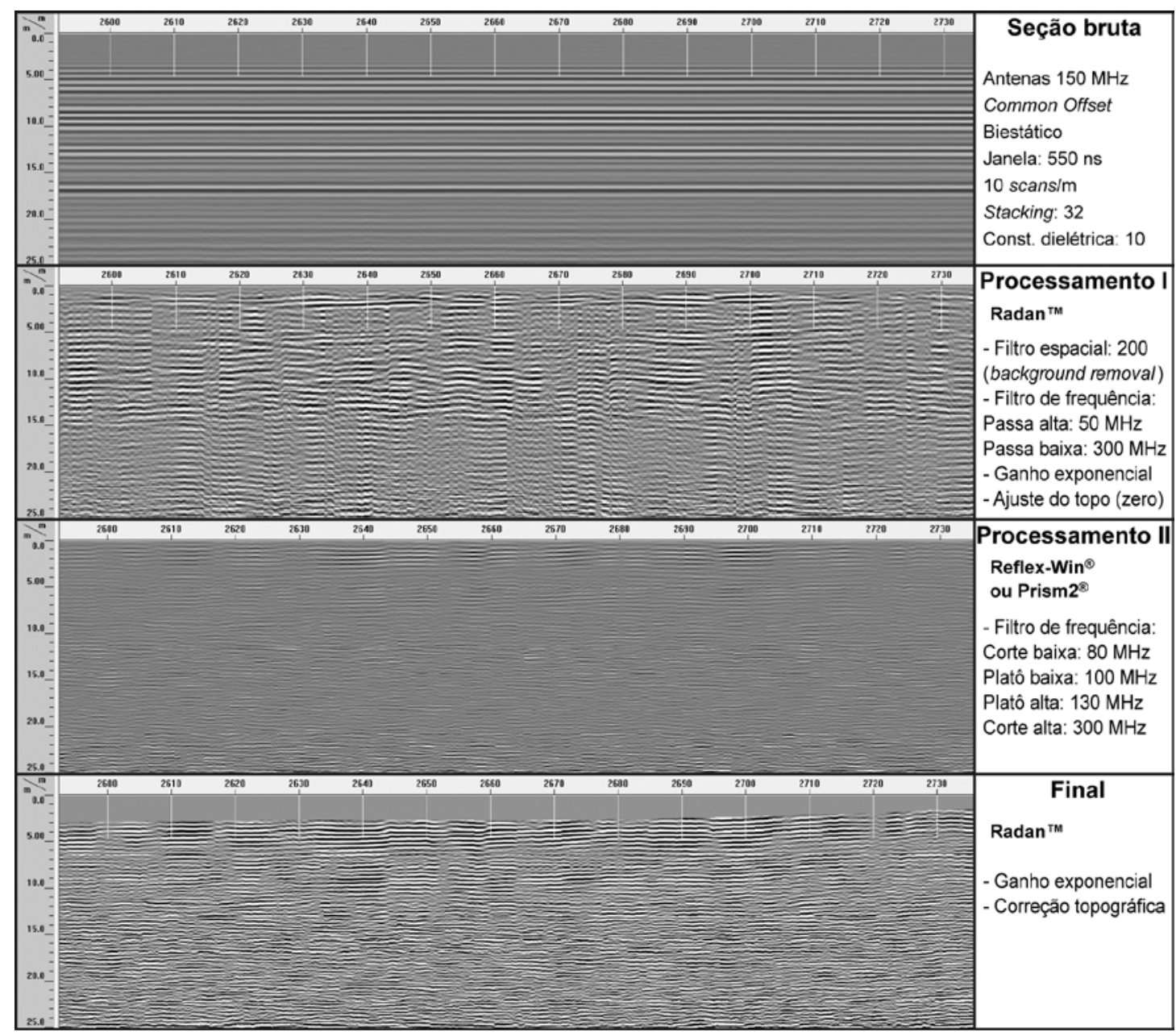

Figura 4 - Exemplo de uma sequência de processamentos aplicada nos dados adquiridos com um arranjo de antenas aéreas no modo bi estático (modificado de Rosa, 2012).

\section{Conclusões}

Após 15 anos de levantamentos de dados ao longo da porção emersa da Bacia de Pelotas foi possível o desenvolvimento de um fluxograma relacionado tanto à aquisição, quanto ao processamento. Este tem propiciado a obtenção de dados com características e resolução em escala de afloramentos de rochas.

A qualidade dos dados, atrelada à facilidade de aquisição de grande volume de seções, têm possibilitado aos interpretes a identificação das relações geométricas e de preenchimento em sistemas deposicionais costeiros, contribuindo sobremaneira para a parametrização e melhor compreensão da evolução geológica..

\section{Agradecimentos}

Os autores agradecem ao CNPq através do apoio financeiro aos projetos: 472484/06-0; 454804/2008-3, 473227/2010-0; 508477/2010-7; ao apoio em infraestrutura do projeto PETROBRAS/FAURGS 2736-7 e ao termo de cooperação PETROBRAS/UFRGS 0050.0071323.11.9. 


\section{Referências}

Barboza, E. G., 1999. Terraços da Margem Leste da Laguna dos Patos, Litoral Médio do Rio Grande do Sul: Estratigrafia e Evolução Holocênica. Programa de PósGraduação em Geociências. Instituto de Geociências. Universidade Federal do Rio Grande do Sul. Porto Alegre-RS. Dissertação de Mestrado. 150p. $<$ http://hdl.handle.net/10183/2865>.

Barboza, E. G., Marques, E. J. J. \& Tomazelli, L. J., 1999. A Utilização do GPR na Identificação de Paleofeições do Registro Estratigráfico no Litoral Médio do Rio Grande do Sul.. In: VII Congresso da Congresso da Associação Brasileira de Estudos do Quaternário, 1999, Porto Seguro-BA. Anais e Guias das Excursões, v. CD, zcp033.pdf.

Barboza, E. G., Rosa, M. L. C. C., Tomazelli, L. J. \& Dillenburg, S. R., 2008. Análise estratigráfica da barreira pleistocênica III através da técnica de georadar na região de Osório - RS. In: $44^{\circ}$ Congresso Brasileiro de Geologia, Curitiba - PR. Anais, 1003-1003.

Barboza, E. G., Dillenburg, S. R., Rosa, M. L. C. C., Tomazelli, L. J. \& Hesp, P. A., 2009. Ground-penetrating radar profiles of two Holocene regressive barriers in southern Brazil. Journal of Coastal Research, SI 56, 579583.

Barboza, E. G., Rosa, M. L. C. C., Dillenburg, S. R. \& Tomazelli, L. J., 2010. The Holocene Coastal Barrier of Rio Grande do Sul (Southern Brazil): an Evaluation Based on GPR Data. In: 2010 Meeting of the Americas. Eos Trans. AGU, Meet. Am. Suppl., 91, NS11A-03.

Barboza, E. G., Rosa, M. L. C. C., Hesp, P. A., Dillenburg, S. R., Tomazelli, L. J. \& Ayup-Zouain, R. N., 2011. Evolution of the Holocene Coastal Barrier of Pelotas Basin (Southern Brazil) - a new approach with GPR data. Journal of Coastal Research, SI 64, 646-650.

Barboza, E. G., Rosa, M. L. C. C., Aguilar, C. G. \& Soldateli, A., 2012. Aplicação de georadar nas dunas do cabo Polônio (Uruguai). In: $46^{\circ}$ Congresso Brasileiro de Geologia, Santos-SP. Anais= Proceedings. São Paulo: Sociedade Brasileira de Geologia, v. CD.

Barboza, E. G., Rosa, M. L. C. C., Dillenburg, S. R. \& Tomazelli, L. J., 2013. Preservation potential of foredunes in the stratigraphic record. Journal of Coastal Research, SI 65, 1265-1270.

Barboza, E. G., Rosa, M. L. C. C., Dillenburg, S. R. Biancini da Silva, A. \& Tomazelli, L. J., 2014a. Stratigraphic analysis applied on the recognition of the interface between marine and fluvial depositional systems. Journal of Coastal Research, SI 70, 687-692.

Barboza, E. G., Rosa, M. L. C. C., Dillenburg, S. R., Ayup-Zouain, R. N., Tomazelli, L. J. \& Biancini da Silva, A., 2014b. Evolução da Barreira Costeira Holocênica na
Região do Estreito - Litoral Médio do Rio Grande do Sul/Brasil. In: XIX Congreso Geológico Argentino, 2014, Córdoba - Argentina. Actas, v. CD - S12-3.

Becker, J. E. G., Barboza, E. G., Dillenburg, S. R. \& Stevaux, R. S., 2008. Uso de GPR Aliado a Dados de Furo de Sondagem para Caracterizar a Evolução da Barreira Costeira Holocênica no Balneário de Dunas

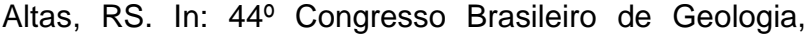
Curitiba - PR. Anais, v. 1, 1021-1021.

Biancini da Silva, A., Barboza, E. G., Rosa, M. L. C. C. \& Fracalossi, F. G., 2010. Caracterização dos Depósitos Sedimentares em Subsuperfície no Setor Meridional da Planície Costeira Sul de Santa Catarina. Gravel, v. 8, p. 1-7.

Biancini da Silva, A., Barboza, E. G., Martinho, C. T. \& Rosa, M. L. C. C., 2013. Análise Estratigráfica de um Paleocanal Holocênico na Barreira Regressiva SulCatarinense. In: XIV Congresso da Associação Brasileira de Estudos do Quaternário, 2013, Natal-RN. Resumos de Trabalhos, v. CD.

Biancini da Silva, A., Barboza, E. G., Rosa, M. L. C. C. \& Dillenburg, S. R., 2014. Meandering Fluvial System Influencing the Evolution of a Holocene Regressive Barrier in Southern Brazil. Journal of Coastal Research, SI 70, 205-210.

Bitencourt, V. J. B., Dillenburg, S. R., Caron, F. \& Barboza, E. G., 2013. Caracterização Morfológica e Estratigráfica das Cristas de Praia da Lagoa dos Quadros, Rio Grande do Sul, Brasil - Resultados Preliminares. In: XIV Congresso da Associação Brasileira de Estudos do Quaternário, Natal-RN. Resumos de Trabalhos, 2013. v. CD.

Bitencourt, V. J. B., Dillenburg, S. R., Barboza, E. G., Caron, F., Manzolli, R. P. \& Biancini da Silva, A., 2014. Morfologia e Estratigrafia de uma Planície de Cristas de Praia Lacustres no Litoral Norte do Rio Grande do Sul, Brasil. In: XIX Congreso Geológico Argentino, 2014, Córdoba - Argentina. Actas, v. CD - S12-5.

Caron, F., 2014. Estratigrafia e evolução da barreira holocênica na Região Costeira de Santa Vitória do Palmar, Planície Costeira do Rio Grande do Sul, Brasil. Programa de Pós-Graduação em Geociências. Instituto de Geociências. Universidade Federal do Rio Grande do Sul. Porto Alegre-RS. Tese de Doutorado. 167p. $<$ http://hdl.handle.net/10183/88625>.

Caron, F., Lima, L. G., Dillenburg, S. R., Tomazelli, L. J., Barboza, E. G., Antiqueira, J. A. F., Rosa, M. L. C. C., Manzolli, R. P. \& Silva, A.B., 2010. Morphology, stratigraphy and factors controlling evolution of a transgressive barrier in southern Brazil. In: 2010 Meeting of the Americas. Eos Trans. AGU, Meet. Am. Suppl., 91, OS21A-01. 
Caron, F., Tomazelli, L. J., Lima, L. G., Barboza, E. G., Rosa, M. L. C. C. \& Dillenburg, S. R., 2011. Características Estratigráficas da Barreira Trangressiva Holocênica na Praia dos Concheiros, Planície Costeira do Rio Grande do Sul, Brasil. In: XIII Congresso da Associação Brasileira de Estudos do Quaternário ABEQUA, 2011, Armação de Búzios - RJ. Anais, v. CD, 107-111.

Caron, F., Barboza, E. G., Tomazelli, L. J., Rosa, M. L. C. C. \& Dillenburg, S. R., 2014. Fases de Transgressão/Regressão na Barreira Costeira Holocênica na Região de Santa Vitória do Palmar, Rio Grande do Sul, Brasil. In: XIX Congreso Geológico Argentino, 2014, Córdoba - Argentina. Actas, v. CD - T1-10.

Davis, J.L \& Annan, A.P., 1989. Ground penetrating radar for high resolution mapping of soil and rock stratigraphy. Geophysical Prospecting, 37, 531-551.

Dillenburg, S. R. \& Barboza, E. G., 2014. The Dip and Strike-Fed Sandy Coast of Southern Brazil. In: Martini, I. P. \& Wanless H. R. (eds.). Sedimentary Coastal Zones from High to Low Latitudes: Similarities and Differences, Geological Society, London, Special Publications 388, doi:10.1144/SP388.16.

Fornari, M., Giannini, P. C. F. \& Nascimento Junior, D. R., 2012. Facies associations and controls on the evolution from a coastal bay to a lagoon system, Santa Catarina Coast, Brazil. Marine Geology, 323-325, 56-68.

Fracalossi, F. G., Barboza, E. G., Rosa, M. L. C. C. \& Silva, A. B., 2010. O Registro em Subsuperfície da Barreira Pleistocênica entre Osório e Tramandaí - RS. Gravel, 8, 21-31.

Jol, H. M. \& Bristow, C. S., 2003. GPR in sediments: advice on data collection, basic processing and interpretation, a good practice guide. In: Bristow, C. S. \& Jol, H. M. (eds.). Ground Penetrating Radar in Sediments. Geological Society, London Special Publications, 211, 0927.

Lima, L. G., Dillenburg, S. R., Medeanic, S., Barboza, E. G., Rosa, M. L. C. C., Tomazelli, L. J., Dehnhardt, B. A. \& Caron, F., 2013. Sea-level rise and sediment budget controlling the evolution of a transgressive barrier in southern Brazil. Journal of South American Earth Sciences, 42, 27-38.

Manzolli, R. P., Portz, L. C., Rosa, M. L. C. C., Barboza, E. G. \& Tomazelli, L. J., 2010. A Utilização de GPR e Sensoriamento Remoto na Identificação e Determinação da Taxa de Acresção de Beach Ridges Lagunares na Margem Oeste da Laguna Lagoa dos Patos. In: Anais do IV Congresso de Oceanografia, v. CD.

Oliveira, J. F. \& Barboza, E. G., 2014. A Dinâmica Costeira no Litoral de Jaguaruna, Santa Catarina. In: XIX Congreso Geológico Argentino, 2014, Córdoba Argentina. Actas, v. CD - S12-17.
Rockett, G. C., Barboza, E. G. \& Rosa, M. L. C. C., 2013. Estimativa da Espessura Sedimentar e Relações Estratigráficas na Região da Planície de Deflação do Campo de Dunas de Itapeva/RS, através de dados de Georradar. In: XIV Congresso da Associação Brasileira de Estudos do Quaternário, Natal-RN. Resumos de Trabalhos, v. CD.

Rockett, G. C., Barboza, E. G., Rosa, M. L. C. C. \& Gruber, N. L.S., 2014. Caracterização Geológica e Geomorfológica do Campo de Dunas de Itapeva, RS, Brasil. In: XIX Congreso Geológico Argentino, 2014, Córdoba - Argentina. Actas, v. CD - S12-36.

Rosa, M. L. C. C., 2012. Geomorfologia, padrões de empilhamento e potencial de preservação do registro estratigráfico dos sistemas deposicionais costeiros no Quaternário do Rio Grande do Sul. Programa de PósGraduação em Geociências. Instituto de Geociências. Universidade Federal do Rio Grande do Sul. Porto Alegre-RS. Tese de Doutorado. 246p. <http://hdl.handle.net/10183/66367>.

Rosa, M. L. C. C., Tomazelli, L. J. \& Barboza, E. G., 2012. Caracterização através de dados de georadar da barreira costeira entre o balneário de Nova Tramandaí e Jardim do Éden, litoral norte do Rio Grande do Sul. In: $46^{\circ}$ Congresso Brasileiro de Geologia, Santos-SP. Anais= Proceedings. São Paulo: Sociedade Brasileira de Geologia, v. CD.

Salgado, E. T., Tomasi Neto, D., Portz, L. C., Manzolli, R. P., Barboza, E. G. \& Tomazelli, L. J., 2013. Identificação da Ocorrência de Washover no trecho Sul da Margem Oeste da Laguna dos Patos através de Imagens de GPR. In: XIV Congresso da Associação Brasileira de Estudos do Quaternário, 2013, Natal-RN. Resumos de Trabalhos, v. CD.

Sangree, J. B. \& Widmier, J. M., 1979. Interpretation of depositional facies from seismic data. Geophysics, 44, 131-160.

Tomazelli, L. J., Barboza, E. G., Dillenburg, S. R., Rosa, M. L. C. C., Caron, F. \& Lima, L. G., 2008. Implantação, preenchimento e desenvolvimento de vales incisos na porção sul da Planície Costeira do Rio Grande do Sul. Projeto PETROBRAS. Relatório Interno, 102p.

Villwock, J. A. \& Tomazelli, L. J., 1995. Geologia costeira do Rio Grande do Sul. Notas Técnicas. CECO, Instituto de Geociências, UFRGS, Porto Alegre, 8, 45p.

Watanabe, D. S. Z., Barboza, E. G., Rosa, M. L. C. C., Martins, E. M. \& Ayup-Zouain, R. N., 2013. Caracterização Geométrica do Acoplamento das Barreiras Pleistocênicas no Litoral Sul do Rio Grande do Sul, Brasil. In: XIV Congresso da Associação Brasileira de Estudos do Quaternário, 2013, Natal-RN. Resumos de Trabalhos, v. CD. 